\title{
O exercício na insuficiência cardíaca: da contra-indicação à evidência científica
}

A insuficiência cardíaca crônica (ICC) pode ser definida como uma situação clínica na qual o débito cardíaco é incapaz de satisfazer a demanda metabólica dos tecidos da periferia. Esta anormalidade desencadeia uma série de mecanismos compensatórios e, desta forma, a insuficiência cardíaca não é mais vista simplesmente como um déficit da função sistólica ou diastólica, mas também como uma síndrome neurohumoral. Em uma fase mais avançada, estas alterações se tornam deletérias e o prognóstico é inversamente proporcional ao grau de ativação neuroendócrina ${ }^{1}$. A ICC apresenta uma incidência anual nos Estados Unidos de aproximadamente 2.000.000 casos. É o diagnóstico de alta hospitalar mais comum nos indivíduos acima de 65 anos de idade e a taxa de mortalidade em cinco anos é freqüentemente superior à de pacientes portadores de diversos tipos de neoplasias ${ }^{2}$.

A intolerância ao esforço e às atividades do cotidiano e a dispnéia são os sintomas mais freqüentes e debilitantes nos pacientes com ICC leve a moderada. Tradicionalmente, sempre houve uma recomendação para que os pacientes com ICC se mantivessem tanto quanto possível em repouso, evitando a realização de qualquer tipo de atividade física que fosse capaz de desencadear sintomas. Assim, sempre se evitou submeter estes pacientes a um teste de esforço ou recomendar atividade física ou reabilitação ${ }^{3}$. De fato, até recentemente a atividade física regular não fazia parte das recomendações para o tratamento dos pacientes com $\mathrm{ICC}^{4,5}$.

Os pacientes com ICC representam um grupo um tanto quanto heterogêneo em relação às anormalidades que determinam a redução da sua capacidade funcional. Há vários subgrupos de anormalidades musculares e hemodinâmicas, que podem se superpor em cada paciente. Há pacientes com fluxo muscular normal ou alterado durante o exercício; nos pacientes com alterações de fluxo, parece haver algum grau de disfunção endotelial. Há pacientes que possuem uma atrofia muscular por alterações intrínsecas das fibras musculares - neste caso uma provável etiologia seria o drive simpático cronicamente aumentado; em outros pacientes, a atrofia muscular seria meramente por inatividade física, com alterações comuns a indivíduos sedentários sem doença; em alguns outros pacientes, a desnutrição contribuiria para a atrofia muscular. De qualquer forma, o elemento comum a todos os pacientes é uma insuficiência muscular esquelética ${ }^{6,7}$, que seria o principal responsável pela redução da capacidade funcional.

A atividade física regular induz várias adaptações morfofuncionais nos pacientes com ICC. Estas adaptações, do ponto de vista qualitativo, são semelhantes às observadas em indivíduos aparentemente saudáveis e atletas. Os estudos da literatura, com algumas óbvias variações conforme o foco da avaliação, mostram achados até certo ponto semelhantes, que incluem: aumentos variáveis no consumo máximo de oxigênio, no consumo de oxigênio e na carga de trabalho nos quais se atinge o limiar anaeróbico, na capacidade de realizar e manter um determinado trabalho mecânico; redução da $\mathrm{FC}$ em repouso e para esforços submáximos; aumento no tamanho das fibras musculares, na quantidade e no volume das mitocôndrias, bem como na atividade das enzimas oxidativas ${ }^{8-10}$. O aumento da capacidade funcional induzido pelo exercício regular está associado com um melhor prognóstico ${ }^{11}$.

Além disso, dois achados particularmente interessantes nos pacientes com ICC e recentemente demonstrados são a melhora da disfunção autonômica, com redução do tônus simpático e aumento do tônus vagal cardíaco ${ }^{12}$, e a reversão da disfunção endotelial presente em muitos desses pacientes ${ }^{13}$. Todos esses achados disponíveis na literatura até o ano passado, aliados à 
experiência de serviços de reabilitação cardíaca em todo o mundo, não deixavam dúvida que o exercício físico se constituía em uma intervenção extremamente benéfica para o paciente portador de ICC sob vários pontos de vista e que presumivelmente também traria um impacto positivo em termos de prognóstico.

As tão esperadas evidências vieram neste ano, com um interessante estudo randomizado e controlado $^{14}$, no qual 50 pacientes com ICC foram submetidos a um programa de 14 meses de duração que incluiu exercícios em cicloergômetro a $60 \%$ do $\mathrm{VO}_{2}$ máximo; os resultados foram comparados com os de um grupo controle com 49 pacientes, que não foram submetidos a programas formais de exercícios. Os autores demonstraram uma redução importante e inequívoca na mortalidade e na freqüência de reinternações, além de uma melhora expressiva da qualidade de vida e de outros parâmetros já demonstrados por estudos anteriores.

Este artigo certamente é o primeiro de uma série e vem corroborar uma conduta já preconizada por muitos já há muito tempo. Desta forma, há agora um irrefutável argumento científico a favor da prática regular de exercícios no paciente portador de ICC. Aliás, esses pacientes estão entre os que mais se beneficiam de programas formais de reabilitação cardíaca e ignorar esse aspecto equivale cientificamente a negligenciar o próprio tratamento farmacológico.

\title{
REFERÊNCIAS
}

1. Cohn JN, Rector TS. Prognosis of congestive heart failure and predictors of mortality. Am J Cardiol 1988;62:25A.

2. Minotti JR, Dudley G. Pathophysiology of exercise intolerance and the role of exercise training in congestive heart failure. Current Opinion in Cardiology 1993;8:397-403.

3. Piña IL, Fitzpatrick JT. Exercise and heart failure - a review. Chest 1996;110:1317-27.

4. Smith TW, Braunwald E, Kelly RA. The management of heart failure. In: Braunwald E. Heart disease - a textbook of cardiovascular medicine. $4^{\text {th }}$ ed. Philadelphia: WB Saunders, 1992:464-519.

5. ACC/AHA Task Force Report on Practice Guidelines. Guidelines for the evaluation and management of heart failure report of the American College of Cardiology/American Heart Association Task Force on Practice Guidelines (Committee on Evaluation and Management of Heart Failure). J Am Coll Cardiol 1995;26:1376-98.

6. Drexler H. Skeletal muscle failure in heart failure. Circulation 1992;85:1621-3.

7. Minotti JR, Johnson EC, Hudson TL, Zuroske G, Murata G, Fukushima E, et al. Skeletal muscle response to exercise training in congestive heart failure. J Clin Invest 1990;86:751-8.

8. Sullivan MJ, Higginbotham MB, Cobb FR. Exercise training in patients with severe left ventricular dysfunction - hemodynamic and metabolic effects. Circulation 1988;78:506-15.

9. Belardinelli R, Georgiou D, Cianci G, Purcaro A. Effects of exercise training on left ventricular filling at rest and during exercise in patients with ischemic cardiomyopathy and severe left ventricular systolic dysfunction. Am Heart J 1996;132: $61-70$.

10. Meyer K, Schwaibold M, Westbrook S, Beneke R, Hajric R, Görnandt L, et al. Effects of short-term exercise training and activity restriction on functional capacity in patients with severe chronic congestive heart failure. Am J Cardiol 1996;78: 1017-22.

11. Mancini DM, Eisen H, Kussmaul W, Mull R, Edmunds LH, Wilson JR. Value of peak exercise oxygen consumption for optimal timing of cardiac transplantation in ambulatory patients with heart failure. Circulation 1991;83:778-86.

12. Coats AJS, Adamopoulos S, Radaelli A, McCance A, Meyer TE, Bernardi L, et al. Controlled trial of physical training in chronic heart failure - exercise performance, hemodynamics, ventilation, and autonomic function. Circulation 1992;85: 2119-31.

13. Hambrecht R, Fiehn E, Weigl C, Gielen S, Hamann C, Kaiser R, et al. Regular physical exercise corrects endothelial dysfunction and improves exercise capacity in patients with chronic heart failure. Circulation 1998;98:2709-15.

14. Belardinelli R, Georgiou D, Cianci G, Purcaro A. Randomized, controlled trial of long-term moderate exercise training in chronic heart failure - effects on functional capacity, quality of life, and clinical outcome. Circulation 1999;99:1173-82.

\author{
José Kawazoe Lazzoli \\ Editor-Chefe, \\ Revista Brasileira de Medicina do Esporte
}


